\title{
Energy-efficient MAC protocol for wireless LANs with WiFi sensors
}

\author{
Woo-Yong Choi ${ }^{1}$
}

\begin{abstract}
More and more mobile computing devices such as smartphones with limited battery power are being used in IEEE 802.11 wireless LANs, and WiFi sensors with very limited battery power are expected to get Internet access through wireless LANs in the near future. We propose an efficient MAC (Medium Access Control) protocol so that WiFi sensors and mobile devices are connected to APs (Access Points) in IEEE 802.11 wireless LANs in an energy-efficient manner.
\end{abstract}

Ke y w ord s: energy efficient MAC protocol, WiFi, LAN, point and distributed coordination function

\section{Introduction}

As our society becomes data-centric, the need for computing devices to be connected to the Internet is growing. IEEE 802.11 wireless LAN technology has the advantage of providing cost-effectively high data rate service in hotspot areas such as homes, schools, shopping malls, and airports. More and more mobile computing devices such as smartphones with limited battery power are being used in wireless LANs, and WiFi sensors, which have very limited battery power, are expected to get the Internet access through wireless LANs. For this reason, we need to develop an energy-efficient MAC (medium access control) protocol for wireless LANs to allow mobile devices and WiFi sensors with low battery power to be connected to APs (access points) in wireless LANs.

The energy-efficient MAC protocols for wireless sensor networks or IEEE 802.11 wireless LANs have been proposed in the literature [1-4]. In [1], the multi-hop communication technique was developed for the nodes deployed in ad hoc manner to be scheduled to go to sleep mode according to applications, and pass application messages through wireless sensor networks. The optimization of the original power saving mechanism in [5], and the IEEE 802.11 ah MAC protocol for WiFi sensors were presented in $[2]$ and $[3,4]$, respectively. However, we still need to resolve the problems of large latency due to multiple transmissions from source to destination in [1], and low transmission rate from WiFi sensors with very limited battery power to APs in [2-4].

In this paper, we propose an efficient MAC protocol for IEEE 802.11 wireless LANs that allows WiFi sensors and mobile devices with limited battery power to indirectly communicate with APs via other nodes with enough transmission power, which are called pseudo APs. Based on the connectivity information between WiFi sensors and mobile devices with limited battery power and other nodes with enough transmission power, to save the energy consumption due to the transmissions of beacon frames of pseudo APs, APs select the minimal number of pseudo APs for WiFi sensors and mobile devices with limited battery power. An pseudo AP and WiFi sensors and mobile devices that are indirectly connected to an AP via the pseudo AP compose a cluster. The hidden node problem can seriously worsen the performance of the DCF (distributed coordination function) protocol, [6]. Each cluster is assigned a separate DCF channel access period during CPs (contention periods) to mitigate the hidden node problem among WiFi sensors and mobile devices with limited transmission power.

\section{Proposed energy efficient MAC protocol}

All nodes should perform the authentication and association processes with APs before they can transmit data frames to and receive from APs. In a wireless LAN, there exist two kinds of nodes that want to be connected to an AP. The first kind of nodes, which are called direct nodes, with enough electric power can directly perform the authentication and association processes with the AP after hearing beacon frames from the AP, and can be directly connected to the AP. The other kind of nodes, which are called indirect nodes, such as WiFi sensors and mobile devices with insufficient battery power cannot be directly connected to the AP with high data rate service. After hearing beacon frames from the pseudo APs that are selected by the AP, the latter kind of nodes indirectly perform the authentication and association processes with the AP through one of the pseudo APs, and are indirectly connected to the AP. The necessary request and response frames for the authentication and association processes between the nodes and the AP are transmitted using the DCF protocol during CPs. The pseudo APs

\footnotetext{
${ }^{1}$ Department of Industrial and Management Systems Engineering, Dong-A University Nakdong-daero, Saha-gu, Busan, Korea, wychoi77@dau.ac.kr
}

https://doi.org/10.2478/jee-2021-0050, Print (till 2015) ISSN 1335-3632, On-line ISSN 1339-309X

(C) This is an open access article licensed under the Creative Commons Attribution-NonCommercial-NoDerivs License (http: //creativecommons.org/licenses/by-nc-nd/3.0/). 
relay the request and response frames for the authentication and association processes between the AP and the indirect nodes.

According to IEEE 802.11 standard in [5], the PCF (point coordination function) and DCF protocols are the fundamental wireless channel access methods, and are respectively operated during CFPs (contention free periods) and CPs, which are alternated with each other. After the authentication and association processes, each direct node can transmit and receive data frames during both CFPs and CPs. However, each indirect node can transmit and receive data frames only during CPs.

Initially each indirect node that wants to be connected to the AP broadcasts the hello packet frame including its MAC address to neighboring direct nodes to announce its identity. Each direct node reports to the AP only the change of the MAC addresses of the indirect nodes that it can hear. Maintaining the set Si of the MAC addresses of the indirect nodes that can be heard, each direct node $\mathrm{i}$ reports to the $\mathrm{AP}$ the $\mathrm{MAC}$ addresses of the indirect nodes that it can newly hear, and the MAC addresses of the indirect nodes that it has not heard any longer during ten consecutive CPs by piggybacking the MAC addresses on its data frames destined for the AP.

Based on the reported connectivity information between the direct nodes and the indirect nodes, the AP selects the appropriate pseudo APs, and let them broadcast their beacon frames. After hearing beacon frames from the pseudo APs, each indirect node selects one of the pseudo APs, and performs the authentication and association processes with the AP through the selected pseudo AP. Beacon frames broadcast from the pseudo APs contain the change of the MAC addresses of the indirect nodes for which they can function as the pseudo APs, that is, the MAC addresses of the new indirect nodes for which they can function as the pseudo APs, and the MAC addresses of the old indirect nodes for which they can no longer function as the pseudo APs. Even though multiple direct nodes can function as the pseudo APs for a specific indirect node as announced by beacon frames of the pseudo APs, the indirect node should select only one direct node as its pseudo AP.

After the successful indirect authentication and association processes, the indirect nodes are allowed to transmit their data frames to and receive from the pseudo APs using the DCF protocol during CPs. The pseudo APs relay data frames between the AP and the indirect nodes. When the pseudo APs successfully receive data frames from the AP or the indirect nodes, the pseudo APs transmit ACK (acknowledgement) frames to the AP or the indirect nodes. Successfully receiving data frames from the pseudo APs, the AP and the indirect nodes transmit ACK frames to the pseudo APs. When the direct nodes detect the change of the connectivity between themselves and the indirect nodes during the course of the transmissions of data frames from the indirect nodes, the change of the connectivity is reported to the AP similarly to the aforementioned method.
The AP maintains the set of selected pseudo APs. When one or more direct nodes are newly selected as the pseudo APs or are removed from the set of pseudo APs, to notify this change to the corresponding direct nodes, the AP broadcasts beacon frames including the MAC addresses of the direct nodes added to or removed from the set of pseudo APs. In case the indirect nodes can no longer receive beacon frames from their pseudo APs due to the change of the set of pseudo APs selected by the AP, they can look for new pseudo APs hearing beacon frames from other pseudo APs, or can broadcast the hello packet frames, and let the AP select the pseudo APs appropriate for them.

Each cluster of a pseudo AP and the indirect nodes that are indirectly connected to the AP via the pseudo AP is assigned a separate DCF channel access period during CPs. This helps us mitigate the hidden node problem among the indirect nodes with insufficient transmission power. CPs consist of the access periods for the direct nodes, and the separate access periods for the clusters of the pseudo APs and the indirect nodes. The AP can access the wireless channel in every access periods of the direct nodes and the clusters using the DCF protocol during CPs. The information of the time of the start of each access period is contained in beacon frames broadcast from the AP and the corresponding pseudo APs.

To save the energy consumption due to the transmissions of beacon frames of the pseudo APs, we need to derive the minimal number of direct nodes, among which each indirect node can find at least one direct node that can function as its pseudo AP. The optimization problem for deriving the minimal number of pseudo APs can be formulated as the set cover problem, where the goal is to find the minimal number of sets Si's whose union equals to the union of Si's of all direct nodes [7]. Note that Si is the set of the MAC addresses of the indirect nodes that can be heard by direct node $i$. The greedy heuristic can be employed to get a good solution for the set cover problem, [7].

\section{Simulation results}

It is assumed that over a circular IEEE 802.11 ah wireless LAN service area, 100 nodes with sufficient transmission power, and $n=1000,2000, \ldots, 5000 \mathrm{WiFi}$ sensors with insufficient battery power are uniformly distributed. The AP is located at the center of the service area. All nodes are assumed to continuously attempt to transmit their data frames with payloads of 5000 bits. All nodes can directly transmit their data frames to the AP. However, when it comes to the direct transmission rates from the nodes to the AP, there is the imbalance that the data transmission rate from WiFi sensors with insufficient battery power to the AP is significantly lower than the data transmission rate of 100 nodes with sufficient transmission power. 
We want to compare the performances of the IEEE 802.11 ah DCF protocol in [3] with and without the indirect transmission method through the pseudo APs, which is proposed in the previous section and can resolve the imbalance of the transmission rates of the nodes with sufficient and insufficient transmission power. For the indirect transmission method proposed in the previous section, 100 nodes with sufficient transmission power are connected to the $\mathrm{AP}$ as the direct nodes, and each WiFi sensor with insufficient battery power is indirectly connected to the AP through the nearest direct node. This results in 100 clusters of the direct and indirect nodes. For the IEEE 802.11 ah DCF protocol with the proposed indirect transmission method, the MPDU (MAC protocol data unit) aggregation technique is also applied for the pseudo APs to piggyback the aggregated MPDUs of WiFi sensors on their data frames destined for the AP [8]. For fair comparison, the same grouping of the nodes is assumed for the IEEE 802.11 ah DCF protocol without the proposed indirect transmission method to be operated within the RAWs (restricted access windows) during CPs.

For each case of the IEEE 802.11 ah DCF protocol with and without the proposed indirect transmission method and $\mathrm{n}=1000,2000,, 5000$, simulations were conducted during $10^{9}$ time slots. To see the maximal performances of the IEEE 802.11 ah DCF protocol with and without the proposed indirect transmission method, it is assumed that no hidden node problem exists within each cluster, and transmissions fail only due to the collisions of simultaneous transmission attempts from two or more nodes. The values of PHY and MAC parameters used for simulations are shown in Tab. 1.

Table 1. Values of PHY and MAC parameters

\begin{tabular}{lc}
\hline Parameters & Values \\
\hline Trans. rate of direct node & $6.5 \mathrm{M} \mathrm{bps}$ \\
Trans. rate of wifi sensor to AP & $0.65 \mathrm{M} \mathrm{bps}$ \\
Trans. rate of wifi sensor to pseudo AP & $6.5 \mathrm{M} \mathrm{bps}$ \\
Sifs & $160 \mu \mathrm{s}$ \\
Difs & $264 \mu \mathrm{s}$ \\
Time slot & $52 \mu \mathrm{s}$ \\
Phy header trans. Time & $240 \mu \mathrm{s}$ \\
Ack trans. Time & $240 \mu \mathrm{s}$ \\
Minimal contention window & 15 \\
Maximal contention window & 1023 \\
\hline
\end{tabular}

For the IEEE 802.11 ah DCF protocol with and without the proposed indirect transmission method, by simulations we obtained the MAC throughput per direct and WiFi sensor nodes during a separate DCF access period or RAW of CPs as shown in Tab. 2. We can see that the IEEE 802.11 ah DCF protocol with the proposed indirect transmission method has far better MAC throughput than the IEEE 802.11 ah DCF protocol without the proposed indirect transmission method. As the number of WiFi sensors in a cluster increases from 10 to 50, the MAC performances of both the IEEE 802.11 ah DCF protocol with and without the proposed indirect transmission method worsen due to the increased number of collisions among the transmissions of nodes. Even though for a data frame to be transmitted from a WiFi sensor to the AP, two transmissions from the WiFi sensor and a pseudo AP is required by the proposed indirect transmission method, the proposed indirect transmission method is beneficial for the MAC performance because of the increased transmission rate of WiFi sensors.

\section{Conclusions}

We proposed a new MAC protocol for mobile computing devices and WiFi sensors with limited battery power to be accommodated to IEEE 802.11 wireless LANs in an energy-efficient manner. When there is the imbalance of the transmission rates of nodes with sufficient transmission power and mobile computing devices and WiFi sensors with insufficient transmission power, the proposed indirect transmission method allows nodes with insufficient transmission power to transmit their data with high transmission rate to APs through appropriately selected pseudo APs. By simulation results, we can see that the proposed indirect transmission method significantly improves the MAC performance of wireless LANs with WiFi sensors having insufficient battery power.

\section{Acknowledgments}

This work was supported by the Dong-A University research fund.

\section{REFERENCES}

[1] W. Ye, J. Heidemann and D. Estrin, "An Energy-Efficient MAC Protocol for Wireless Sensor Networks", Wireless Sensor Network, vol. 1, pp. 59-69, 2008.

Table 2. MAC throughputs (in kbps) per direct and WiFi sensor nodes

\begin{tabular}{lcccccc}
\hline & & $\mathrm{n}=1000$ & $\mathrm{n}=2000$ & $\mathrm{n}=3000$ & $\mathrm{n}=4000$ & $\mathrm{n}=5000$ \\
\hline DCF without & Direct node & 42.50 & 20.48 & 12.91 & 9.33 & 7.24 \\
proposed method & WiFi sensor & 42.01 & 19.84 & 12.70 & 9.22 & 7.18 \\
DCF with & Direct node & 132.74 & 63.45 & 39.69 & 28.44 & 22.24 \\
proposed method & WiFi sensor & 133.13 & 62.78 & 39.91 & 28.72 & 22.30 \\
\hline
\end{tabular}


[2] E.-S. Jung and N.H. Vaidya, "An Energy Efficient MAC Protocol for Wireless LANs", Proc. of Twenty-First Annual Joint Conference of the IEEE Computer and Communications Societies, 2002.

[3] IEEE Std 802.11 ah, "Wireless LAN Medium Access Control (MAC) and Physical Layer (PHY) Specifications Amendment 2: Sub $1 \mathrm{GHz}$ License Exempt Operation", IEEE Press, 2017.

[4] L. Tian, S. Santi, A. Seferagic, J. Lan and J. Famaey, "Wi-Fi HaLow for the Internet of Things: An Up-to-date Survey on IEEE 802.11 ah Research", Journal of Network and Computer Applications, vol. 182, 2021.

[5] IEEE, IEEE Wireless LAN Edition, IEEE Press, 2003.

[6] W.-Y. Choi, "Fair MAC Protocol for IEEE 802.11 Wireless LANs with Hidden Node Problem", Journal of Electrical Engineering, vol. 71, pp. 365-367, 2020.
[7] R. Hassin and A. Levin, "A Better-than-Greedy Approximation Algorithm for the Minimum Set Cover Problem", SIAM Journal on Computing, vol. 35, pp. 189-200, 2005.

[8] IEEE Std $802.11 \mathrm{n}$, "Wireless LAN Medium Access Control (MAC) and Physical Layer".

Received 12 July 2021

Woo-Yong Choi was born in Busan, Korea 1970. He received the $\mathrm{BS}, \mathrm{MS}$ and $\mathrm{PhD}$ degrees in industrial engineering from POSTECH in 1992, 1994 and 1997, respectively. From 1997 to 2001 he was a senior member of technical staff at Hyundai Electronics Industries Co., Ltd.. From 2001 to 2005 he was a senior member of technical staff at ETRI. Since 2005 he has been with Department of Industrial \& Management Systems Engineering at Dong-A University, where he is currently a full tenured professor. 